\title{
Identification of the WRKY gene family and functional analysis of two genes in Caragana intermedia
}

\author{
Yongqing Wan ${ }^{1 \dagger}$, Mingzhu Mao ${ }^{1 \dagger}$, Dongli Wan ${ }^{2 \dagger}$, Qi Yang ${ }^{1}$, Feiyun Yang ${ }^{3}$, Mandlaa ${ }^{3}$, Guojing Li ${ }^{1} \mathbb{D}$ \\ and Ruigang Wang ${ }^{1 *}$
}

\begin{abstract}
Background: WRKY transcription factors, one of the largest families of transcriptional regulators in plants, play important roles in plant development and various stress responses. The WRKYs of Caragana intermedia are still not well characterized, although many WRKYs have been identified in various plant species.

Results: We identified 53 CiWRKY genes from C. intermedia transcriptome data, 28 of which exhibited complete open reading frames (ORFs). These CiWRKYs were divided into three groups via phylogenetic analysis according to their WRKY domains and zinc finger motifs. Conserved domain analysis showed that the CiWRKY proteins contain a highly conserved WRKYGQK motif and two variant motifs (WRKYGKK and WKKYEEK). The subcellular localization of CiWRKY26 and CiWRKY28-1 indicated that these two proteins localized exclusively to nuclei, supporting their role as transcription factors. The expression patterns of the 28 CiWRKYs with complete ORFs were examined through quantitative real-time PCR (qRT-PCR) in various tissues and under different abiotic stresses (drought, cold, salt, high-pH and abscisic acid (ABA)). The results showed that each CiWRKY responded to at least one stress treatment. Furthermore, overexpression of CiWRKY75-1 and CiWRKY40-4 in Arabidopsis thaliana suppressed the drought stress tolerance of the plants and delayed leaf senescence, respectively.

Conclusions: Fifty-three CiWRKY genes from the C. intermedia transcriptome were identified and divided into three groups via phylogenetic analysis. The expression patterns of the 28 CiWRKYs under different abiotic stresses suggested that each CiWRKY responded to at least one stress treatment. Overexpression of CiWRKY75-1 and CiWRKY40-4 suppressed the drought stress tolerance of Arabidopsis and delayed leaf senescence, respectively. These results provide a basis for the molecular mechanism through which CiWRKYs mediate stress tolerance.
\end{abstract}

Keywords: WRKY gene family, Caragana Intermedia, Abiotic stress, Gene expression, Subcellular localization, Function analysis

\section{Background}

Transcription factors are a class of proteins that significantly regulate plant growth and development and play an important role in plant defense and stress responses $[1,2]$. WRKY transcription factors are one of the largest families of transcriptional regulators in plants [2]. These transcription factors contain the WRKY domain, which

\footnotetext{
* Correspondence: wangruigang@imau.edu.cn

${ }^{\dagger}$ Equal contributors

${ }^{1}$ College of Life Sciences, Inner Mongolia Key Laboratory of Plant Stress Physiology and Molecular Biology, Inner Mongolia Agricultural University, Hohhot, China

Full list of author information is available at the end of the article
}

is a region of 60 amino acids that is highly conserved [3]. The WRKY domain comprises a featured WRKYGQK sequence motif and a zinc finger-like motif [4]. The WRKY sequence is occasionally replaced by WRRY, WSKY, WKRY, WVKY or WKKY in a subset of WRKY proteins [5]. The zinc finger structure is either $\mathrm{CX}_{4-5} \mathrm{CX}_{22-23} \mathrm{HXH} \quad\left(\mathrm{C}_{2} \mathrm{H}_{2}\right.$-type $)$ or $\mathrm{CX}_{7} \mathrm{CX}_{23} \mathrm{HXC}$ ( $\mathrm{C}_{2} \mathrm{HC}$-type) [5]. At least one WRKY domain is present in all known WRKY proteins [3].

WRKY proteins can be classified into three main groups (group I, group II and group III) according to the number of WRKY domains along with features of the 
zinc finger-like motif [3]. Group I proteins have two WRKY domains: an N-terminal WRKY domain and a Cterminal WRKY domain, with $\mathrm{C}_{2} \mathrm{H}_{2}$ zinc finger type $\mathrm{CX}_{4} \mathrm{CX}_{22-23} \mathrm{HXH}$. Group II proteins harbor a single WRKY domain and a $\mathrm{C}_{2} \mathrm{H}_{2}$-type $\mathrm{CX}_{4-5} \mathrm{CX}_{23} \mathrm{HXH}$ zinc finger. Group III proteins contain a single WRKY domain and a $\mathrm{C}_{2} \mathrm{HC}$-type $\mathrm{CX}_{7} \mathrm{CX}_{23} \mathrm{HXC}$ zinc finger [3]. Group II WRKY proteins were further divided into five subgroups on the basis of their primary amino acid sequences: IIa, IIb, IIc, IId and IIe [5]. Furthermore, WRKY proteins specifically bind to the W box $(\mathrm{C} /$ T)TGAC $(\mathrm{T} / \mathrm{C})$ DNA sequence $[3,4]$, and this binding requires both WRKY and zinc finger domains [4].

The first 4 WRKY cDNAs (SPF1, ABF $(1,2), P_{c} W R K Y$ $(1,2,3)$ and $Z A P 1)$ were cloned from sweet potato (Ipomoea batatas), wild oat (Avena fatua), parsley (Petroselinum crispum) and Arabidopsis thaliana, respectively [3]. Numerous WRKY proteins have been studied in various plant species. For example, there are 72 identified members in A. thaliana [6], 182 in soybean (Glycine max) [7], 127 in apple (Malus domestica Borkh.) [8], 80 in grape (Vitis vinifera) [9], 86 in Brachypodium distachyon [10], 85 in cassava (Manihot esculenta) [11], 95 in carrot (Daucus carota) [12], 61 in Salvia miltiorrhiza [1], 103 in rice (Oryza sativa) [13], and 32 in Broomcorn millet (Panicum miliaceum L.) [14]. In maize (Zea mays), 136 WRKY proteins encoded by 119 WRKY genes have been identified [15]. In cotton, 116 and 102 WRKY genes have been identified from Gossypium raimondii and Gossypium hirsutum [16], respectively.

WRKY transcription factors are involved in multiple plant processes, such as seed development $[17,18]$, dormancy [19] and germination [20], trichome development [21], root development [22], leaf senescence [23], plant growth and flowering [24, 25]. Notably, WRKYs have been broadly reported to be involved in plant defense responses. For instance, 49 out of 72 WRKYs from $A$. thaliana are differentially expressed after pathogen infection or SA treatment [6], and A. thaliana WRKYs including AtWRKY7, 11, 17, 18, 23, 25, 27, 38, 40, 41, $48,53,58,60$, and 62 serve as negative regulators of defense signaling [26]. OsWRKY62 and OsWRKY76 of rice negatively regulate defense upon pathogen infection [27]. Overexpression of OsWRKY13 [28] and OsWRKY45-2 [29] in rice increases resistance to bacterial blight and fungal blast caused by Xanthomonas oryzae pv. Oryzae and Magnaporthe grisea, respectively, while overexpression of OsWRKY42 decreases rice resistance to $M$. oryzae [30]. The transcriptional regulatory cascade of WRKY13, WRKY42, and WRKY45-2 is necessary for defending rice against fungal pathogens [30]. In cotton (G. hirsutum L.), GhWRKY15 is involved in disease resistance [31]. Overexpression of grape $V v W R K Y 1$ in tobacco results in enhanced resistance to various fungi [32]. The roles of WRKYs in plant responses to abiotic stresses such as drought [33-36], cold [37], salinity [37], heat [38, 39], low Pi [40, 41], ultraviolet B (UV-B) [42], osmotic stress [43], and ABA [33, 36, 44] have been widely reported. These studies suggested that WRKYs are involved in multiple stress responses.

Caragana intermedia, a native desert perennial shrub belonging to the Leguminosae family, is distributed in sandy grasslands and desert regions of west and northwest China and Mongolia, and is widely valued due to its high forage value and sand-fixing capacity, along with its strong resistance to drought and salinity [45-48]. C. intermedia is considered to be an ideal plant for elucidating the mechanisms of abiotic stress tolerance [49], and research on its molecular mechanisms has been increasing in recent years. For instance, to normalize quantitative real-time PCR (qRT-PCR) data from $C$. intermedia under different abiotic stress conditions, suitable reference genes were screened, which was useful for further gene expression analysis [50]. The transcription of two NAC transcription factors, CiNAC3 and CiNAC4, was found to be induced under treatment with $A B A$ and various abiotic stresses, and ectopic expression of CiNAC3 and CiNAC4 in Arabidopsis reduces the inhibition of seed germination by $\mathrm{ABA}$ and enhances the salt tolerance of the transgenic plants [51]. The expression patterns of miR2118 and its target genes (CiDR1 and CiDR2) from $C$. intermedia are altered under drought stress, and constitutive overexpression of cin-miR2118 in tobacco enhances the plant's tolerance to drought stress [46]. Abiotic stresses $\left(\mathrm{NaCl}, \mathrm{ZnSO}_{4}, \mathrm{CdCl}_{2}\right.$, high/low temperature, and dehydration) induce the expression of the glutamate decarboxylase-encoding genes CiGAD1 and CiGAD2, except for CiGAD2 under Cd stress, and ABA has been shown to be involved in regulating the expression of CiGADs in response to salt stress [52]. However, there is limited knowledge of the function of WRKYs in C. intermedia. In this study, we identified 53 CiWRKY genes based on transcriptomic data from $C$. intermedia, performed phylogenetic analysis and WRKY domain alignment, and examined the subcellular localizations of two CiWRKYs and the expression patterns of 28 of CiWRKYs in different tissues and under various abiotic stresses. Furthermore, we overexpressed CiWRKY75-1 and CiWRKY40-4 in A. thaliana and characterized their function. This work provides a basis for exploring the molecular roles of WRKYS and facilitates further investigation of the molecular mechanisms of abiotic stress tolerance in C. intermedia.

\section{Results}

Identification and cloning WRKY genes from C. intermedia To identify WRKYS in the drought-treated transcriptome of $C$. intermedia, the sequences of the $A$. thaliana 
WRKY family were used to query homologous $C$. intermedia sequences. After Blast searches against the sequences in NCBI using blastX to predict conserved domains and remove redundant sequences, 53 sequences with apparent WRKY domains were annotated as C. intermedia WRKY genes. Among these genes, 21 WRKYs with complete open reading frames (ORFs) were identified. Using the rapid-amplification of $c D N A$ ends (RACE) technique, an additional 7 WRKYS were cloned, and their full-length ORFs were obtained. The ORF lengths of these 28 genes ranged from $489 \mathrm{bp}$ to $2229 \mathrm{bp}$, and their amino acid lengths ranged from 163 to 743 aa (details are provided in Table 1). The 28 WRKYS with complete ORFs were used for further expression analysis.

\section{Phylogenetic analysis and multiple sequence alignment of WRKY domains}

To further analyze the evolution of these CiWRKYs, we constructed a phylogenetic tree based on a total of 140 WRKYs (72 from A. thaliana, 53 from C. intermedia and 15 from G. max distributed in different groups) (Fig. 1). According to the number of WRKY domains and the pattern of zinc finger structures, the 53 CiWRKY proteins were distributed in three main groups. Eleven CiWRKYs were assigned to group I. Thirty-four CiWRKYs were distributed in group II, which was further classified into five sub-groups: IIa, IIb, IIc, IId, and IIe, which contained 5, 6, 13, 4 and 6 CiWRKYs, respectively. The remaining 8 CiWRKYs belonged to group III.

Table 1 Characteristics of WRKY genes in C. intermedia

\begin{tabular}{|c|c|c|c|c|c|c|c|}
\hline Gene name & cDNA length & $\mathrm{Aa}$ & $\mathrm{Mw}(\mathrm{KDa})$ & Group & WRKY domain & Zinc finger & Arabidopsis ortholog \\
\hline CiWRKYZ2 & 2229 & 743 & 80.46 & Group I & WRKYGQK×2 & $\mathrm{CX}_{4} \mathrm{CX}_{22-23} \mathrm{HXH}$ & AtWRKY2 \\
\hline CiWRKY3-1 & 1383 & 461 & 50.31 & Group I & WRKYGQK×2 & $\mathrm{CX}_{4} \mathrm{CX}_{22-23} \mathrm{HXH}$ & AtWRKY3 \\
\hline CIWRKYY3-2 & 1536 & 512 & 55.86 & Group I & WRKYGQK×2 & $\mathrm{CX}_{4} \mathrm{CX}_{22-23} \mathrm{HXH}$ & AtWRKY3 \\
\hline CiWRKY6-1 & 1656 & 552 & 59.97 & Group Ilb & WRKYGQK & $\mathrm{CX}_{5} \mathrm{CX}_{23} \mathrm{HXH}$ & AtWRKY6 \\
\hline CiWRKY6-2 & 1830 & 610 & 65.56 & Group IIb & WRKYGQK & $\mathrm{CX}_{5} \mathrm{CX}_{23} \mathrm{HXH}$ & AtWRKYG \\
\hline CIWRKY12 & 696 & 232 & 26.32 & Group Ilc & WRKYGQK & $\mathrm{CX}_{4} \mathrm{CX}_{23} \mathrm{HXH}$ & AtWRKY12 \\
\hline CiWRKY15 & 1005 & 335 & 36.43 & Group Ild & WRKYGQK & $\mathrm{CX}_{5} \mathrm{CX}_{23} \mathrm{HXH}$ & AtWRKY15 \\
\hline CIWRKY17 & 1017 & 339 & 36.59 & Group IId & WRKYGQK & $\mathrm{CX}_{5} \mathrm{CX}_{23} \mathrm{HXH}$ & AtWRKY17 \\
\hline CiWRKY23 & 957 & 319 & 35.71 & Group Ilc & WRKYGQK & $\mathrm{CX}_{4} \mathrm{CX}_{23} \mathrm{HXH}$ & AtWRKY23 \\
\hline CIWRKY26 & 1722 & 574 & 63.28 & Group I & WRKYGQK×2 & $\mathrm{CX}_{4} \mathrm{CX}_{22-23} \mathrm{HXH}$ & AtWRKY26 \\
\hline CIWRKY28-1 & 999 & 333 & 36.98 & Group IIc & WRKYGKK & $\mathrm{CX}_{4} \mathrm{CX}_{23} \mathrm{HXH}$ & AtWRKY 28 \\
\hline CIWRKY $28-2$ & 975 & 325 & 37.19 & Group IIC & WRKYGQK & $\mathrm{CX}_{4} \mathrm{CX}_{23} \mathrm{HXH}$ & AtWRKY28 \\
\hline CiWRKY30 & 1083 & 361 & 40.79 & Group III & WRKYGQK & $\mathrm{CX}_{7} \mathrm{CX}_{23} \mathrm{HXC}$ & AtWRKY30 \\
\hline CIWRKY32-1 & 1521 & 507 & 54.68 & Group I & WRKYGQK×2 & $\mathrm{CX}_{4} \mathrm{CX}_{22-23} \mathrm{HXH}$ & AtWRKY32 \\
\hline CIWRKY33-1 & 1614 & 538 & 59.57 & Group I & WRKYGQK×2 & $\mathrm{CX}_{4} \mathrm{CX}_{23} \mathrm{HXH}$ & AtWRKY33 \\
\hline CIWRKY36 & 1785 & 595 & 64.36 & Group Ilb & WRKYGQK & $\mathrm{CX}_{5} \mathrm{CX}_{23} \mathrm{HXH}$ & AtWRKY36 \\
\hline CIWRKY40-1 & 990 & 330 & 36.78 & Group Ila & WRKYGQK & $\mathrm{CX}_{5} \mathrm{CX}_{23} \mathrm{HXH}$ & AtWRKY40 \\
\hline CiWRKY 40-3 & 825 & 275 & 30.97 & Group Ila & WRKYGQK & $\mathrm{CX}_{5} \mathrm{CX}_{23} \mathrm{HXH}$ & AtWRKY40 \\
\hline CiWRKY $40-4$ & 897 & 299 & 33.66 & Group Ila & WRKYGQK & $\mathrm{CX}_{5} \mathrm{CX}_{23} \mathrm{HXH}$ & AtWRKY40 \\
\hline CIWRKY41-1 & 1083 & 361 & 41.12 & Group III & WRKYGQK & $\mathrm{CX}_{7} \mathrm{CX}_{23} \mathrm{HXC}$ & AtWRKY41 \\
\hline CiWRKY48 & 1155 & 385 & 42.15 & Group Ilc & WRKYGQK & $\mathrm{CX}_{4} \mathrm{CX}_{23} \mathrm{HXH}$ & AtWRKY48 \\
\hline CIWRKYY 50 & 489 & 163 & 19.22 & Group IIc & WRKYGKK & $\mathrm{CX}_{4} \mathrm{CX}_{23} \mathrm{HXH}$ & AtWRKY50 \\
\hline CIWRKY51 & 558 & 186 & 21.55 & Group Ilc & WRKYGKK & $\mathrm{CX}_{4} \mathrm{CX}_{23} \mathrm{HXH}$ & AtWRKY51 \\
\hline CiWRKY56 & 678 & 226 & 25.61 & Group IIc & WRKYGQK & $\mathrm{CX}_{4} \mathrm{CX}_{23} \mathrm{HXH}$ & AtWRKY56 \\
\hline CiWRKY57 & 834 & 278 & 30.68 & Group IIC & WRKYGQK & $\mathrm{CX}_{4} \mathrm{CX}_{23} \mathrm{HXH}$ & AtWRKY57 \\
\hline CiWRKY69-1 & 798 & 266 & 28.89 & Group lle & WRKYGQK & $\mathrm{CX}_{5} \mathrm{CX}_{23} \mathrm{HXH}$ & AtWRKY69 \\
\hline CIWRKY70-1 & 939 & 313 & 35.24 & Group III & WRKYGQK & $\mathrm{CX}_{7} \mathrm{CX}_{23} \mathrm{HXC}$ & AtWRKY70 \\
\hline CiWRKY75-1 & 567 & 189 & 21.08 & Group IIc & WRKYGQK & $\mathrm{CX}_{4} \mathrm{CX}_{23} \mathrm{HXH}$ & AtWRKY75 \\
\hline
\end{tabular}




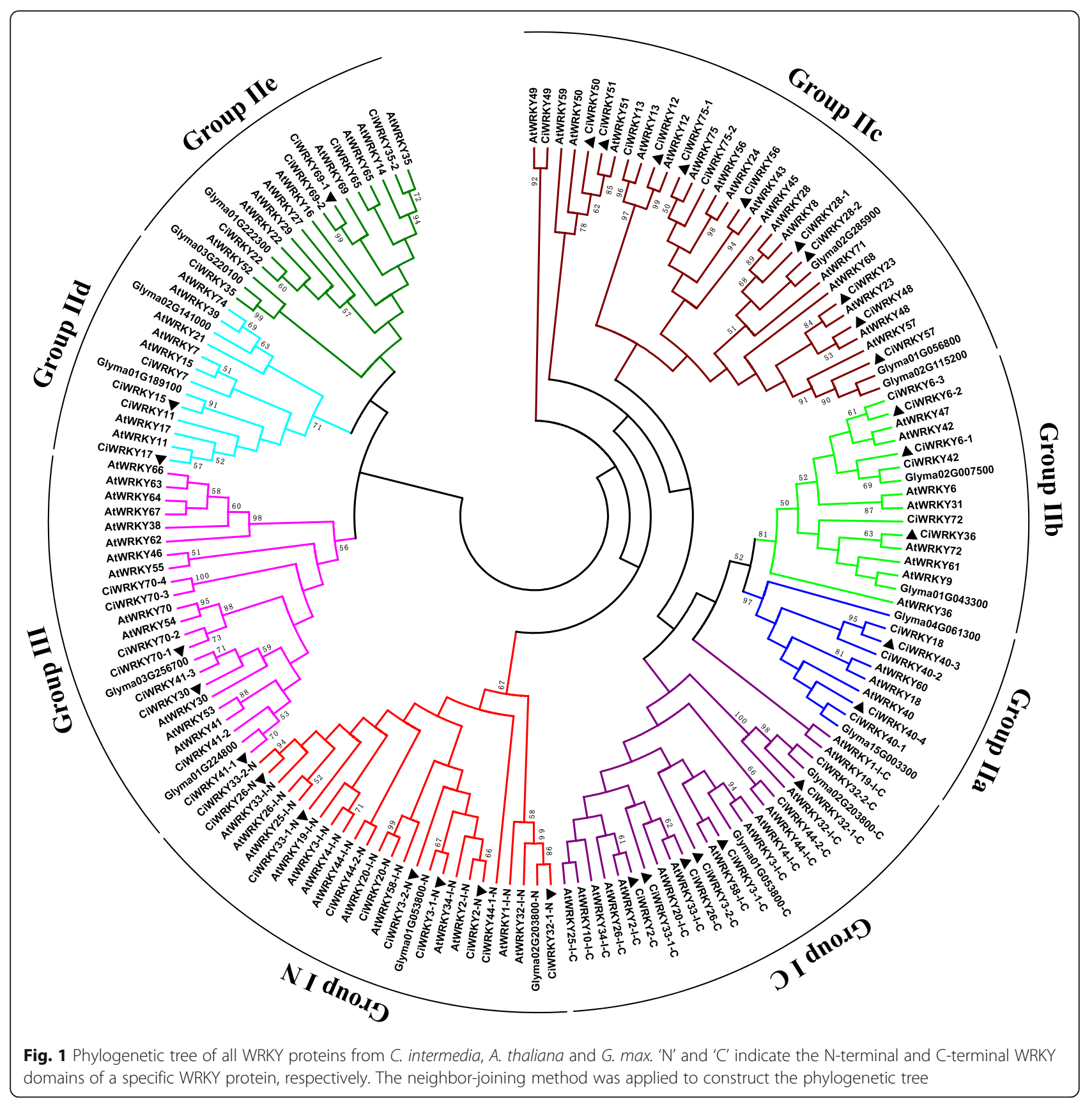

Multiple sequence alignments of the core domains of the CiWRKYs showed that, in group I, with the exception of CiWRKY44-1, which exhibited a WKKYEEK variant sequence, all others harbored the conserved WRKYGQK sequence, and all displayed the apparent $\mathrm{C}_{2} \mathrm{H}_{2}$-typezinc finger $\left(\mathrm{CX}_{4} \mathrm{CX}_{22-23} \mathrm{HXH}\right)$. Among the 34 group II proteins containing one WRKY domain, 32 CiWRKYs carried the conserved WRKYGQK sequence, while CiWRKY50 and CiWRKY51 exhibited the WRKYGKK sequence instead. The $\mathrm{C}_{2} \mathrm{H}_{2}$-type zinc finger $\left(\mathrm{CX}_{4-5} \mathrm{CX}_{23} \mathrm{HXH}\right)$ was observed in 33 CiWRKYs. Seven of the eight group III WRKYs harbored the WRKYGQK sequence, while the variant sequence WRKYGKK was present in CiWRKY41-2. The other group III CiWRKYs exhibited $\mathrm{C}_{2} \mathrm{HC}$-type zinc finger $\left(\mathrm{CX}_{7} \mathrm{CX}_{23} \mathrm{HXC}\right)$ with the exception of CiWRKY41-3 (Fig. 2).

\section{Subcellular localization of CiWRKYs}

To examine the subcellular localization of the CiWRKY proteins, one of the proteins containing two WRKY domains (CiWRKY26) and one of the proteins containing one-WRKY-domain (CiWRKY28-1) were fused with green fluorescent protein (GFP). The roots of the T2 generation of 35S::CiWRKYs-GFP transgenic Arabidopsis 


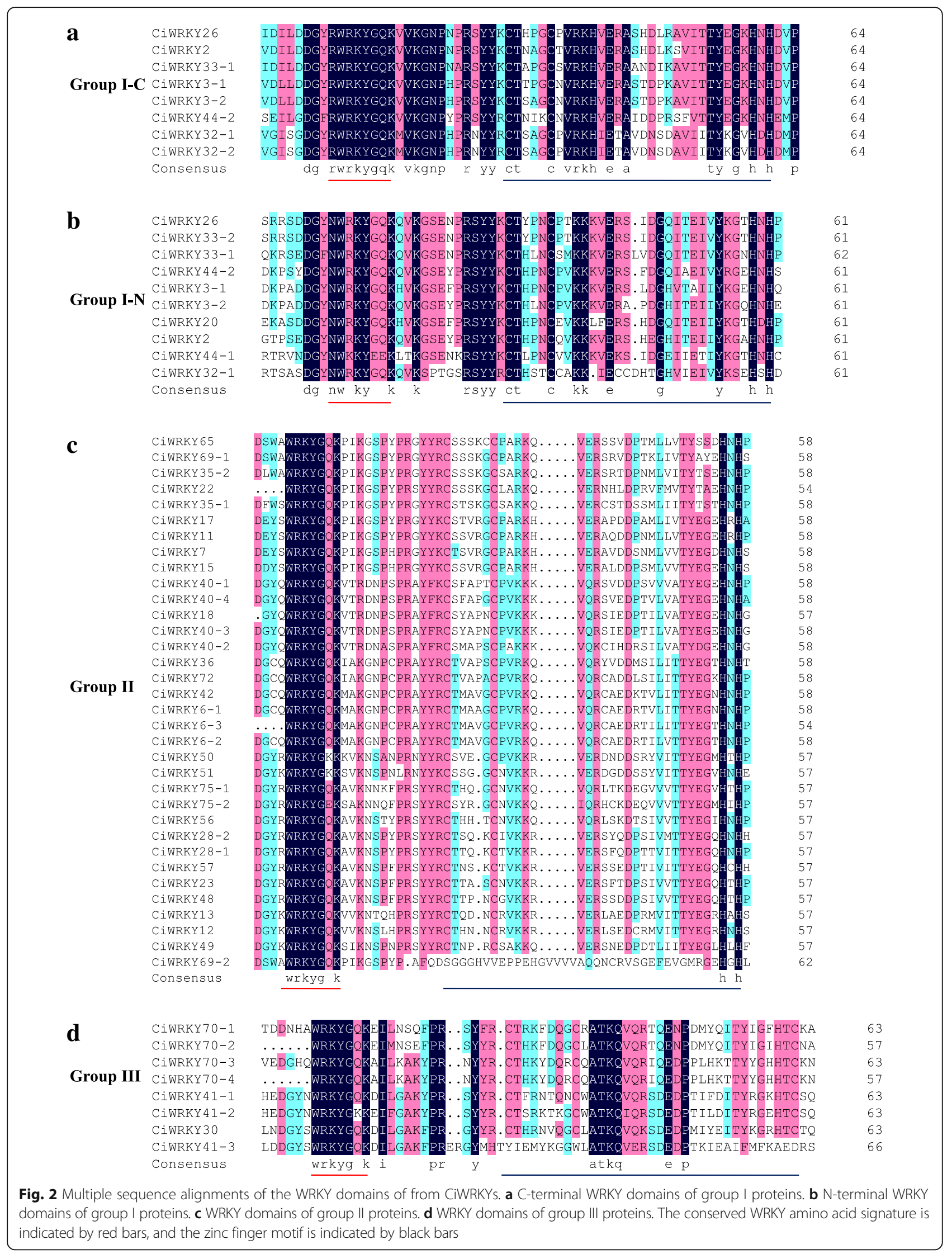


seedlings were used to examine GFP activity. GFP fluorescence was exclusively observed in the nuclei of the transgenic Arabidopsis seedlings, while GFP was ubiquitously present throughout the cells of the 35S::GFP transgenic lines (Fig. 3). The nuclear localization of these two CiWRKYs is in agreement with their putative roles as transcription factors.

\section{Expression patterns of CiWRKYs in C. intermedia}

The expression patterns of the 28 CiWRKYs with complete ORFs in wild C. intermedia tissues, including the roots, stems and leaves, were examined via qRTPCR. As shown in Fig. 4 and Additional file 1: Table S1, four genes (CiWRKY3-2, CiWRKY6-2, CiWRKY23 and CiWRKY26) exhibited relatively high expression levels in all examined tissues, especially in the leaves. CiWRKY321, CiWRKY17 and CiWRKY15 were also ubiquitously expressed, but at relatively low levels compared with the above four genes. In contrast, the transcripts of eight genes, including CiWRKY36, CiWRKY75-1, CiWRKY12, CiWRKY50, CiWRKY28-1, CiWRKY48, CiWRKY40-4 and CiWRKY6-1, were detectable, but showed extremely low abundance in all tissues. CiWRKY28-2 and CiWRKY56 were mainly expressed in stems, while CiWRKY40-1 and CiWRKY30 were predominantly found in leaves. CiWRKY69-1 showed higher transcript abundance in the roots than in other tissues. The other CiWRKY genes showed lower expression globally, and most of them exhibited lower transcript abundance in the roots than in the leaves or stems.

\section{Expression patterns of CiWRKYs under abiotic stresses}

Malondialdehyde (MDA), proline (PRO) and soluble total sugar (SS) are indicators of plant physiological status. As shown in Additional file 2: Figure S1, drought treatment induced the accumulation of MDA, PRO and SS, whose levels peaked at $48 \mathrm{~h}$. These findings indicated that the applied drought treatment was effective.

The expression patterns of the 28 of CiWRKYs were further assessed using qRT-PCR, under drought treatment as well as other abiotic stresses, including salt, abscisic acid (ABA), cold, and high-pH treatments (Table 2). The gene expression of twenty-four CiWRKYs genes was found to be induced by drought treatment, with changes of 2- to 149-fold. In contrast, the expression of CiWRKY33-1, CiWRKY15 and CiWRKY69-1 was inhibited by drought treatment, and no obvious change in the expression of CiWRKY32-1 was observed (Fig. 5). Salt treatment down-regulated the expression of 18 CiWRKYS and up-regulated the expression of 5 CiWRKYs (Additional file 3: Figure S2). Under ABA treatment, the expression of $22 \mathrm{CiWRKYs}$ was increased, while that of CiWRKY3-1 and CiWRKY33-1 was decreased, and CiWRKY2, 3-2, 32-1 and 69-1 were not obviously affected (Additional file 4: Figure S3). Under cold treatment, the expression of 14 CiWRKYs was upregulated, and the transcripts of 11 CiWRKYs were down-regulated. The expression of three other CiWRKYs presented no change compared with the expression seen in the control (Additional file 5: Figure S4). High-pH treatment enhanced the expression of 12 CiWRKYs.

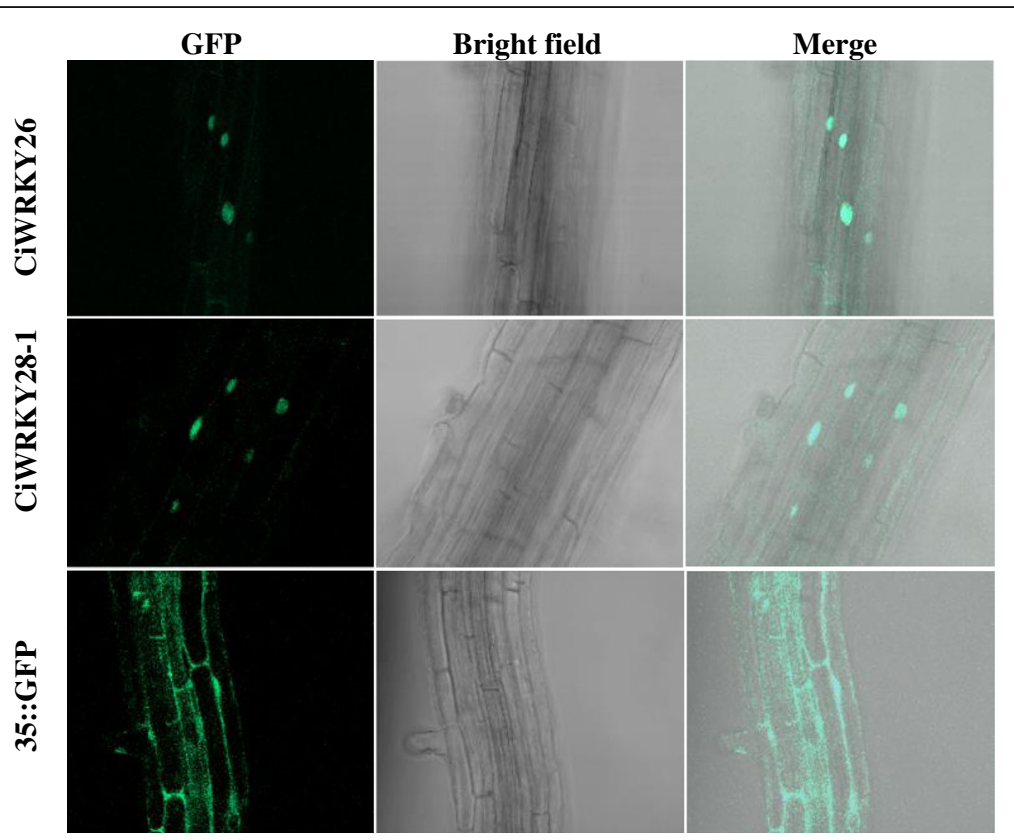

Fig. 3 Subcellular localization of two C. intermedia WRKYs. The roots of 35S::CiWRKY26-GFP (upper panel), 355::-CiWRKY28-1-GFP (middle panel), and 35S:: GFP (bottom panel) transgenic plants were used for observations of GFP fluorescence 

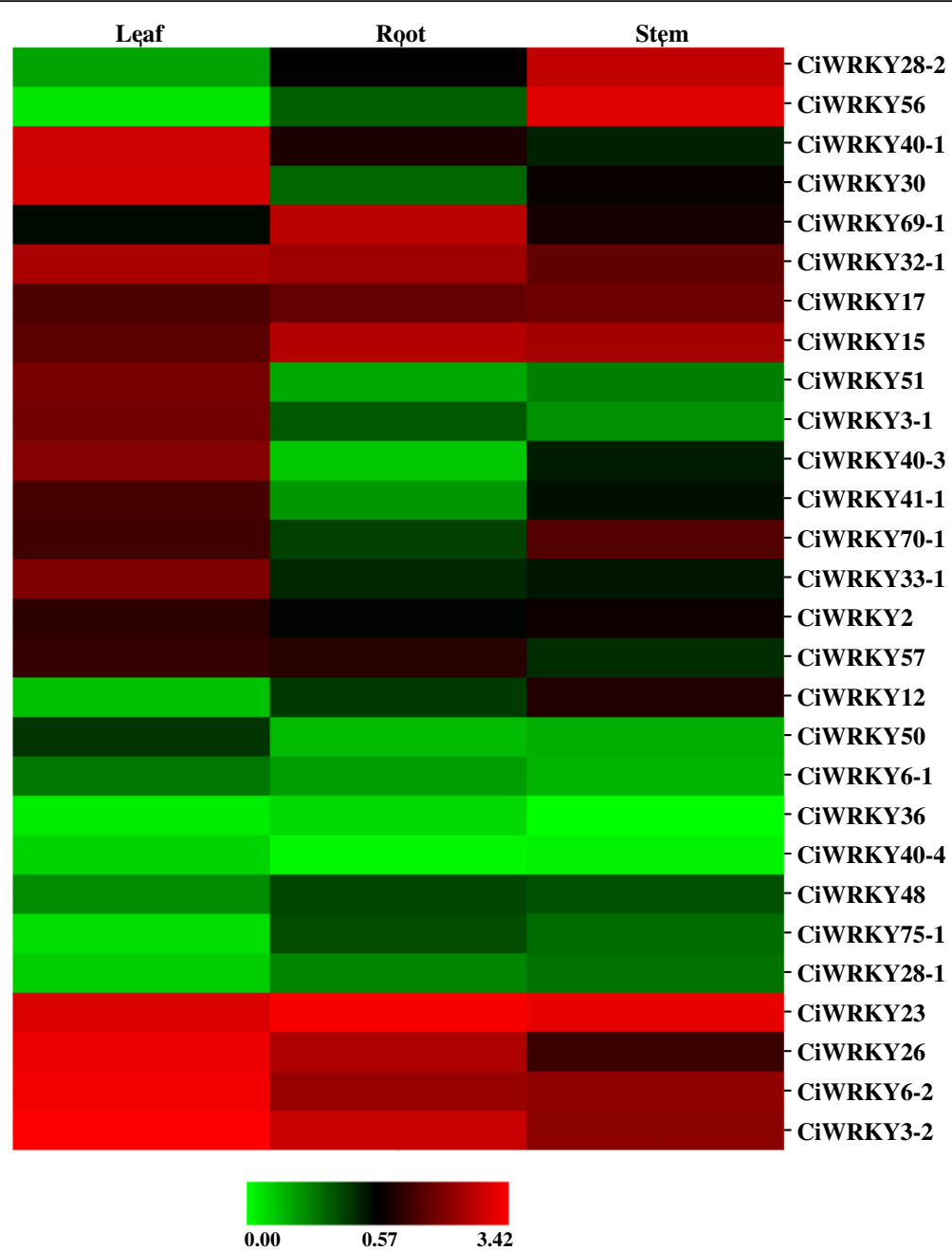

Fig. 4 Expression profiles of CiWRKY genes in different tissues. Samples were collected from the roots $(R)$, stems $(S)$ and leaves $(L)$ of $C$. intermedia. The transcripts of 28 CiWRKYS with full-length sequences were examined via qRT-PCR. The relative expression values were calculated using the $2^{-\Delta C T}$ method, and CiEF1a was employed as the endogenous control. The $2^{-\Delta C T}$-based expression values were used to produce the heatmap

Notably, the maximal expression observed for CiWRKY33-1, $41-1,50$, and 40-4 corresponded to increases of 111-, 365-, 388-, and 109-fold, respectively, whereas the expression of the other CiWRKYs was not affected or even down-regulated after high-pH treatment (Additional file 6: Figure S5). The results indicated that each of these CiWRKY genes responded to at least one stress treatment.

\section{CiWRKY75-1 transgenic Arabidopsis is hypersensitive to drought stress}

CiWRKY75-1, which exhibited relatively high expression under all five treatments (Table 2), was over-expressed in Arabidopsis. Three representative overexpression lines (CiWRKY75-1-OE5, CiWRKY75-1-OE6 and CiWRKY751-OE8) showing relatively high expression levels were used to perform abiotic stress tolerance tests. Four-weekold transgenic Arabidopsis were exposed to drought stress by withholding watering for 15 days and were then re- watered for 3 days. The CiWRKY75-1-overexpressing lines were more sensitive to drought, displaying decreased survival rates and significantly increased MDA levels compared with the wild type (Fig. 6). No morphological differences were observed between the wild-type and CiWRKY75-1-overexpressing plants under normal growth conditions (Fig. 6). These results indicated that overexpression of CiWRKY75-1 reduced the tolerance of Arabidopsis to drought stress, and CiWRKY75-1 acted as a negative regulator in the plant response to drought.

\section{CiWRKY40-4-overexpressing lines show delayed leaf senescence}

According to the observed stress-responsive expression patterns, CiWRKY40-4 was another gene that was induced under all five treatments (Table 2). However, ectopic expression of CiWRKY40-4 in Arabidopsis resulted in no obvious morphological differences from the 
Table 2 Expression patterns of CiWRKYs under different abiotic stresses

\begin{tabular}{|c|c|c|c|c|c|}
\hline Gene name & Drought & Salt & $A B A$ & Cold & High-pH \\
\hline CiWRKYZ & up & $\mathrm{N}$ & $\mathrm{N}$ & down & up \\
\hline CiWRKY3-1 & up & down & down & $\mathrm{N}$ & $\mathrm{N}$ \\
\hline CiWRKY3-2 & up & down & $\mathrm{N}$ & up & $\mathrm{N}$ \\
\hline CiWRKY6-1 & up & down & up & down & $\mathrm{N}$ \\
\hline CiWRKYG-2 & up & up & up & up & up \\
\hline CIWRKY12 & up & down & up & down & down \\
\hline CiWRKY15 & down & down & up & up & up \\
\hline CiWRKY17 & up & down & up & down & down \\
\hline CiWRKY23 & up & $N$ & up & $\mathrm{N}$ & $\mathrm{N}$ \\
\hline CiWRKY26 & up & up & up & up & up \\
\hline CiWRKY28-1 & up & down & up & down & down \\
\hline CiWRKY28-2 & up & $\mathrm{N}$ & up & down & $N$ \\
\hline CiWRKY3O & up & down & up & up & up \\
\hline CiWRKY32-1 & $N$ & down & $\mathrm{N}$ & $N$ & down \\
\hline CiWRKY33-1 & down & down & down & up & up \\
\hline CiWRKY36 & up & down & up & down & $\mathrm{N}$ \\
\hline CIWRKY40-1 & up & up & up & up & up \\
\hline CiWRKY40-3 & up & down & up & up & $\mathrm{N}$ \\
\hline CiWRKY40-4 & up & up & up & up & up \\
\hline CiWRKY41-1 & up & $\mathrm{N}$ & up & up & up \\
\hline CiWRKY48 & up & down & up & down & down \\
\hline CiWRKY50 & up & down & up & down & up \\
\hline CiWRKY51 & up & down & up & down & up \\
\hline CiWRKY56 & up & down & up & down & down \\
\hline CiWRKY57 & up & down & up & up & $\mathrm{N}$ \\
\hline CIWRKY69-1 & down & down & $\mathrm{N}$ & up & $\mathrm{N}$ \\
\hline CiWRKY70-1 & up & $\mathrm{N}$ & up & up & down \\
\hline CiWRKY75-1 & up & up & up & up & up \\
\hline
\end{tabular}

One-month-old $C$. intermedia seedlings were subjected to drought, salt, $A B A$, cold and high-pH treatments, and shoot samples were collected at $0.5,1,3,6$, 12,24 or $48 \mathrm{~h}$. Untreated plants were employed as controls. "Up" indicates up-regulated expression with a minimum 2-fold change compared with the control. "Down" indicates down-regulated expression with a minimum 2-fold change compared with the control. " $\mathrm{N}$ " indicates no obvious change detected

wild type under drought stress. Unexpectedly, under normal growth conditions, CiWRKY40-4-overexpressing Arabidopsis showed delayed leaf senescence compared with the wild type (Fig. 7). To further explore the mechanism of CiWRKY40-4 in leaf senescence, senescence related-genes, including the senescence-associated genes SAG12, SAG13 and SAG29 and the chlorophyll degradation-related genes pheophytinase $(P P H)$, pheophorbide a oxygenase (PAO), non-yellowing1/stay-green1 (NYE1/SGR1), non-yellow coloring1 (NYC1) and NYC1like $(N O L)$ were analyzed. All of these genes were downregulated in the CiWRKY40-4-overexpressing lines compared with the wild type (Fig. 8), indicating that
CiWRKY40-4 delayed plant senescence by negatively regulating senescence-associated gene expression in Arabidopsis.

\section{Discussion}

In this study, we identified 53 CiWRKY-encoding genes from the drought-treated transcriptome database (NCBI Sequence Read Achieve (SRA) accession number SRP121096) based on the presence of WRKY domains [3]. According to the number of their WRKY domains and the features of their zinc finger-like motifs, the CiWRKYs were divided into three groups, as generally described, among which the group II CiWRKYs accounted for the largest proportion of $64 \%$. These findings are consistent with reports regarding the WRKYs found in A. thaliana [3], cassava [11], and soybean [7], which harbor the greatest numbers of group II WRKYs in the family.

Generally, WRKY proteins are defined by the conserved amino acid sequence WRKYGQK [3]; however, this sequence has been replaced by WRRY, WSKY, WKRY, WVKY or WKKY in some WRKY proteins [5]. In the present study, the WKKYEEK variant was observed in CiWRKY44-1 proteins, and the WRKYGKK variant motif replaced the conserved WRKYGQK sequence in CiWRKY41-2, 50 and 51. These findings are consistent with reports describing the WRKY proteins identified in other species, such as $A$. thaliana (AtWRKY51, 52 and 59) [3], V. vinifera L. (VvWRKY8, 13, 14 and 24) [53], and P. miliaceum L. (PmWRKY2, $15,23,24$, and 28) [14].

According to recent reports [54], several of the WRKYs have more than 2 WRKY domains or contain other domains, in addition to the WRKY domains. These other domains include, for example, the ZF_SBP TF domain, kinase domain, PAH domain, ULP_protease domain, TIR domain, LRR domains, NAC domain, ATP_GRASP domain, and B3 domain. Based on these findings, additional novel groups, beyond the three known WRKY groups, were identified [54]. In A. thaliana, Fragaria vesca, Brassica rapa, G. max, G. raimondii, and Sorghum bicolor, 1, 5, 3, 1, 2, and 3 novel WRKY proteins have been found, respectively [54]. We report here that all of the 28 identified CiWRKYs with ORFs contained one or two WRKY domains. In addition to the WRKY domains, NCBI conserved domain database (CDD) annotation [55] showed that CiWRKY36 (Group IIb) contains a DUF972 (domain of unidentified function 972) domain, followed by a WRKY domain, and both CiWRKY15 and CiWRKY17 (Group IId) harbor a plant_Zn_cluster domain, which is located at the $\mathrm{N}$-terminus and is followed by a WRKY domain. CiWRKY6-1 (Group IIb) exhibits a bZIP (basic region/ leucine zipper) domain, followed by a WRKY domain. 


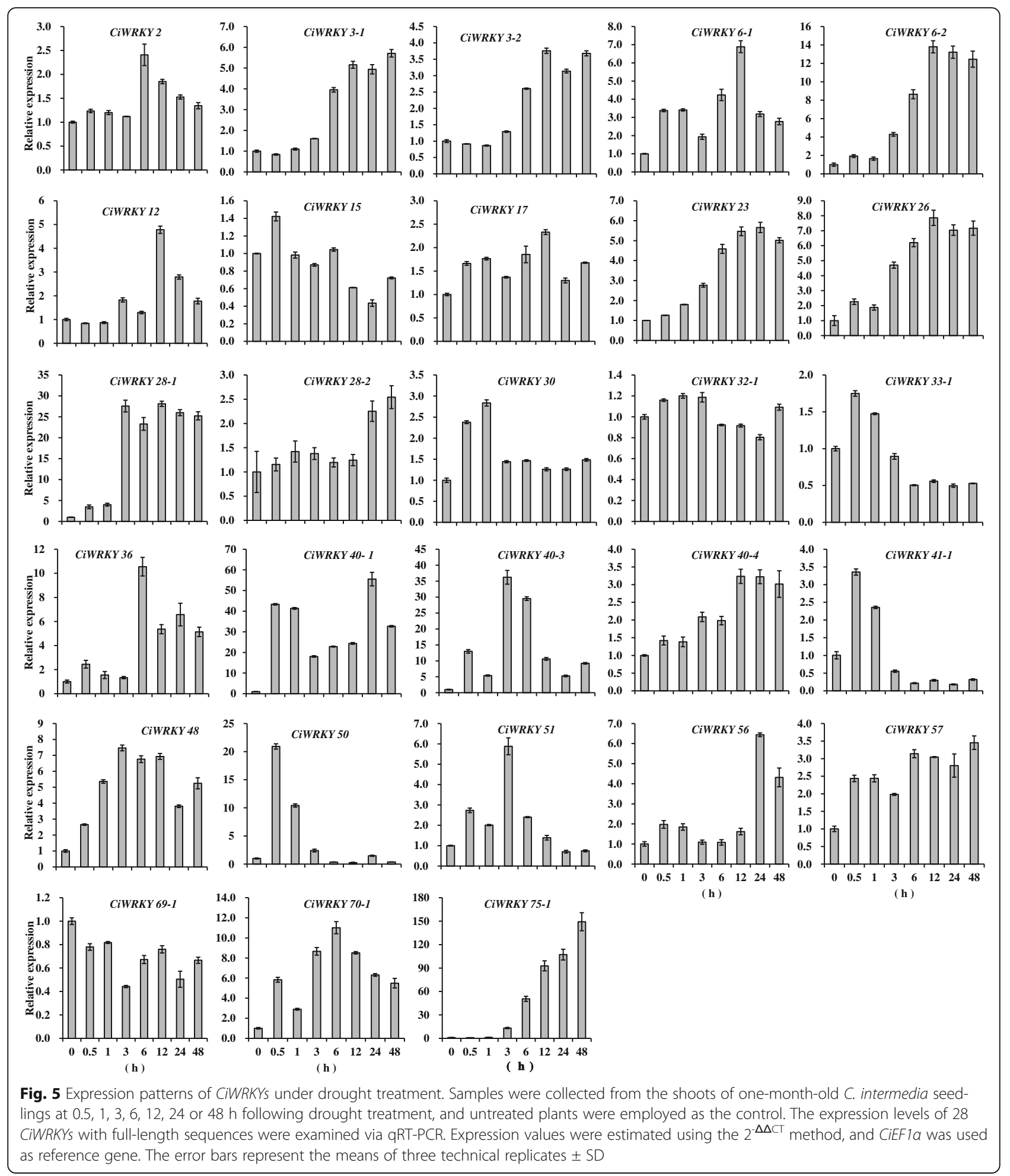

DUF972 belongs to a protein family of unknown function. The plant_Zn_cluster domain is present in two CiWRKYs (CiWRKY15 and CiWRKY17) and is located at the Nterminus of the WRKY domain. Its sequence (EHSDDVSGSGKCHCVKRRKNRVKRTVRVPAISSKIAD) is different from the zinc finger structure, and this domain may have additional functions. bZIP transcription factors are also one of the largest gene families in plants and are involved in multiple plant biological processes, including various stress responses [56, 57]. 

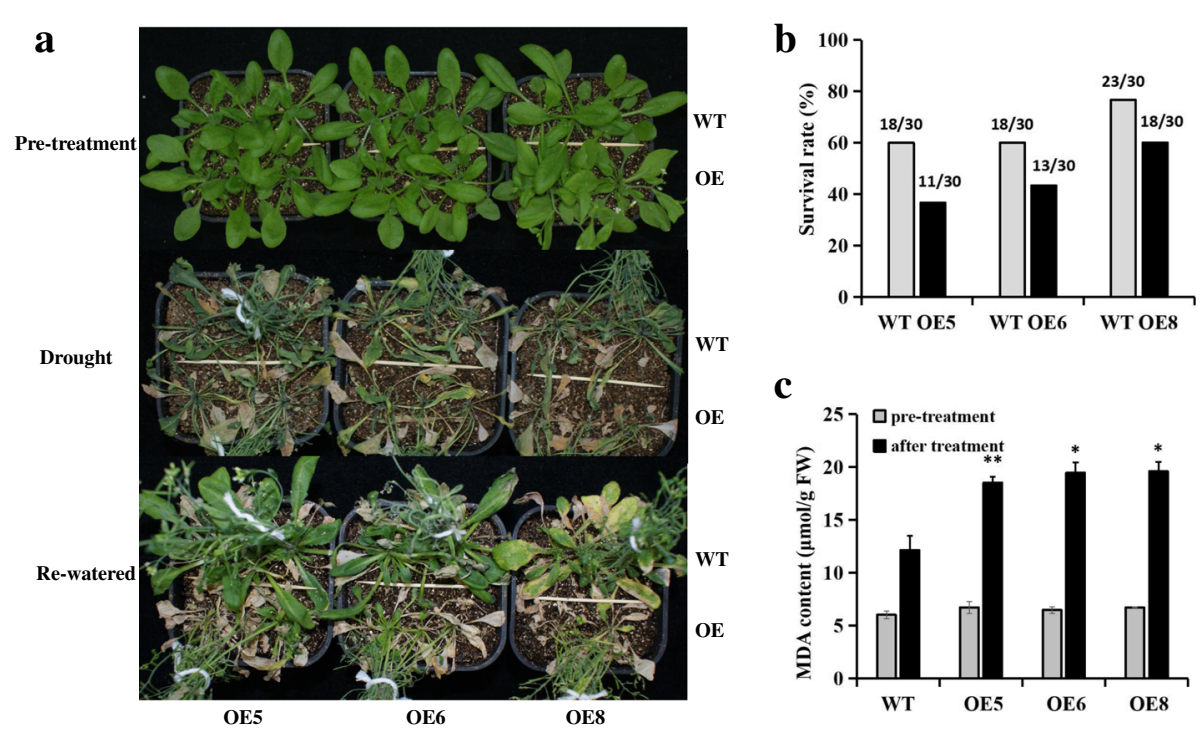

\section{c}

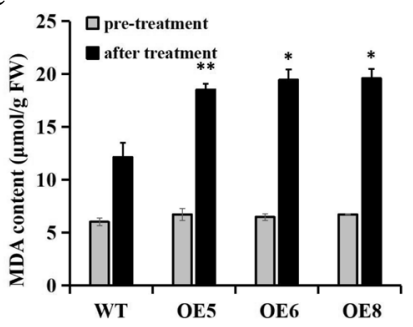

Fig. 6 Transgenic Arabidopsis overexpressing CiWRKY75-1 is hypersensitive to drought stress. Four-week-old CiWRKY75-1 transgenic and wild-type Arabidopsis plants were subjected to drought stress by withholding water for 15 days, followed by re-watering for 2 days. a Photograph of representative plants. $\mathbf{b}$ Calculation of survival rates. Thirty plants from each genotype were used for statistical analysis. $\mathbf{c}$ Measurement of MDA contents. ${ }^{*}$ indicates significant differences $(P<0.05) ;{ }^{*}$ indicates extremely significant differences $(P<0.01)$. OE represents overexpression lines, WT represents wild-type Arabidopsis. The experiments were repeated three times with similar results

Evidence from genome- or transcriptome-wide analyses shows that the response of WRKYS to abiotic stresses varies according to the species. For instance, 30 putative WRKYS were identified through transcriptome analysis in Chinese wild hazel (Corylus heterophylla Fisch), and 23 of these WRKYS were found to be simultaneously up-regulated by cold, drought and high-salinity stresses [58]. Twenty-eight salt-responsive GarWRKYS were identified among 109 WRKY genes in a salt-tolerant wild cotton species (Gossypium aridum) based on transcriptome sequencing data [59]. In P. miliaceum L., 32 WRKYS were identified based on transcriptome analysis, and the expression levels of 22 PmWRKYs were observed to be significantly altered under at least one abiotic stress treatment [14]. Among the 85 WRKY genes identified from the $M$. esculenta genome, 78 were found to be differentially expressed in response to drought stress [11]. In addition, 34 out of 127 WRKYs from the apple genome were identified as differentially expressed under waterlogging stress [8].

Drought is one of the most common environmental stress factors that significantly inhibits plant growth and crop production [60]. The roles of the WRKY transcription factors in the drought response have been widely
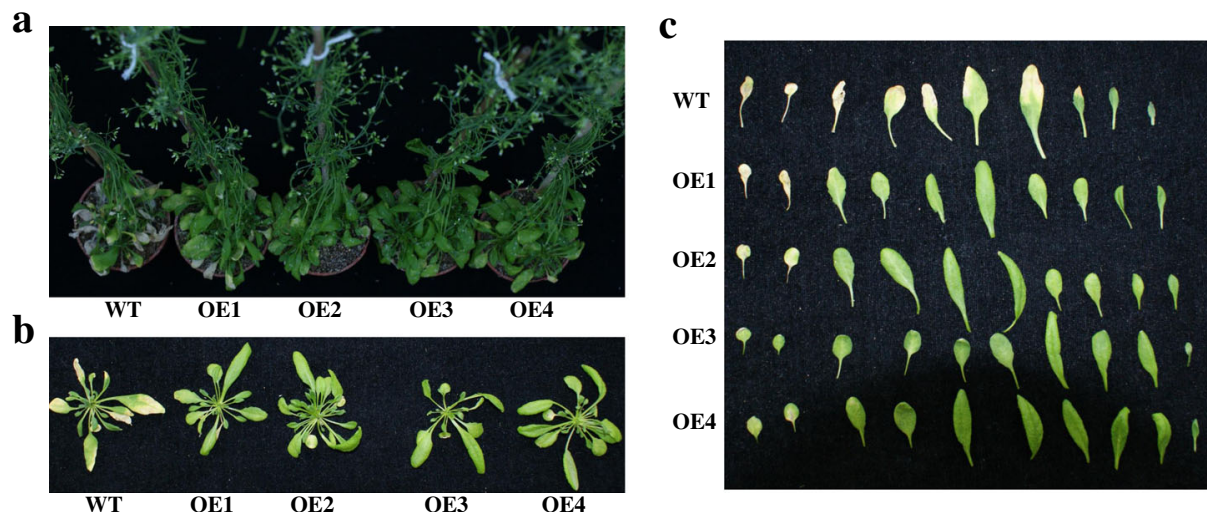

Fig. 7 Overexpression of CiWRKY40-4 delayed Arabidopsis leaf senescence. Six-week-old CiWRKY40-4-overexpressing lines growing in normal conditions exhibited a stay-green phenotype compared with wild-type Arabidopsis. OE represents overexpression lines, WT represents wild-type Arabidopsis. a The adult plants of different genotypes growing under normal conditions. $\mathbf{b}$ The rosette leaves of the plants from $\mathbf{a}$. $\mathbf{c}$ The detached rosette leaves from $\mathbf{b}$ 

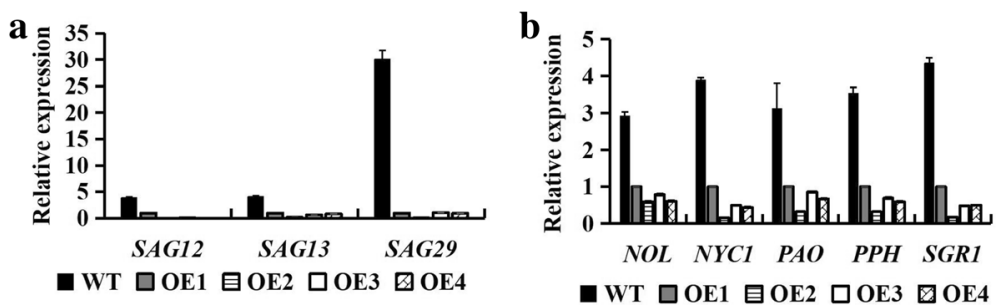

Fig. 8 Expression patterns of senescence-related genes in CiWRKY40-4-overexpressing and wild-type Arabidopsis. Leaf samples were collected from five-week-old CiWRKY40-4-overexpressing lines and wild-type Arabidopsis and were employed for expression analyses through quantitative real-time PCR. a Expression patterns of senescence-associated genes (SAG12, SAG13 and SAG29). b Expression patterns of chlorophyll degradationrelated genes (PPH, PAO, SGR1/NYE1, NOL and NYC1). Expression values were estimated using the $2^{-\triangle \Delta C T}$ method, and AtEF1a was used as the reference gene. The error bars represent the mean of three technical replicates \pm SD. The experiments were repeated three times with similar results

reported. Activated or constitutive expression of WRKY57 confers drought tolerance in A. thaliana [34], and the expression of AtWRKY57 is increased by abiotic stresses such as ABA, dehydration, and mannitol treatment [34]. Overexpression of AtWRKY53 results in hypersensitivity to drought stress by inhibiting stomatal closure [61]. Overexpression of TaWRKY1 and TaWRKY33 in Arabidopsis leads to enhanced plant drought tolerance [62]. WRKY20 transcripts accumulate in wild soybean (G. soja) under ABA, salt, cold, and drought stresses, and overexpression of GsWRKY2O increases the drought tolerance in A. thaliana [33].

We performed a transcriptome analysis to profile gene expression patterns in $C$. intermedia in response to drought stress and identified 53 CiWRKY genes with varying expression patterns in response to drought stress (Additional file 7: Table S2). Further investigation showed that the transcripts of 28 CiWRKYs (with complete ORFs) responded to at least one abiotic stress when plants were subjected to drought, cold, salt, high$\mathrm{pH}$, and ABA treatments. The expression levels of fifteen genes (CiWRKY12, 17, 15, 28-1, 30, 33-1, 40-1, 50, 51, $48,56,26,75-1,40-4$, and 6-2) were altered under all five treatments. Notably, CiWRKY40-1 and CiWRKY751 responded to all treatments intensively. In A. thaliana, AtWRKY75, which is a homologue of CiWRKY75-1 sharing $85 \%$ amino acid sequence similarity, has been reported to regulate $\mathrm{Pi}$ starvation and root development [63] and to play a role in plant resistance to pathogens $[64,65]$. In the present study, the overexpression of CiWRKY75-1 in Arabidopsis compromised the drought stress tolerance of plants. This finding has not been reported by other researchers, and suggests that CiWRKY75-1 serves as a negative regulator under drought stress. Both the mRNA and protein of AtWRKY53 are reported to accumulate in Arabidopsis following drought stress, and AtWRKY53 overexpression enhances hypersensitivity to drought stress compared with that in the wild type, via inhibition of stomatal closure to increase the water loss rate [61]. The AtWRKY46 transcript is also induced by drought stress in Arabidopsis [66], and the AtWRKY46/54/70 signaling complex negatively regulates plant drought tolerance by repressing dehydration-inducible gene expression, which is likely achieved via the simultaneous function of BES1 [67]. However, all four Arabidopsis WRKYs belong to group III, while CiWRKY75-1 belongs to group IIc; thus, the mechanism underlying the regulation of the drought tolerance signaling pathway by CiWRKY75-1, either through direct or indirect interaction with downstream genes, remains unclear. Additionally, no morphological differences during root development were observed between the overexpression lines and wild-type Arabidopsis, and the functions of CiWRKY75-1 in pathogen infection and Pi starvation still require further investigation.

CiWRKY40-4 is in the same group IIa clade as WRKY40, WRKY18, and WRKY60 of A. thaliana and is most similar to AtWRKY40. In A. thaliana, WRKY18, WRKY40 and WRKY60 play partially redundant roles in response to $P$. syringae and $B$. cinerea infection [68]. The wrky18wrky 40 double mutant displays enhanced resistance towards the powdery mildew Golovinomyces orontii. This mutant is also involved in PAMP-triggered basal defense and acts as a negative regulator of positive defense regulators such as CYP71A13, EDS1 and PAD4 [69]. In addition, the expression of WRKY40, WRKY18, and WRKY60 is elevated under $\mathrm{ABA}, \mathrm{NaCl}$ and PEG treatments [44]. The wrky40 knockout mutant of $A$. thaliana exhibits a sensitive phenotype, while WRKY40overexpressing lines show a less sensitive phenotype than the wild type under ABA-induced inhibition of seed germination [44]. WRKY40 represses the ABA-responsive genes, such as $A B I 5$, and functions as negative regulator of in ABA signaling by interacting with ABAR [70]. AtWRKY40 acts as a negative regulator of pathogeninduced cell death regulated by NAC4 [71]. The transcription of WRKY40, WRKY46, WRKY51, WRKY60, WRKY63, 
and WRKY75 is elevated in a loss-of-function mutant of FtSH4, which encodes a mitochondrial protease that is involved in SA-mediated leaf senescence [72]. In the present study, overexpressing CiWRKY40-4 in A. thaliana did not alter drought tolerance but delayed leaf senescence by down-regulating the expression levels of senescencerelated genes. Senescence is the final step in leaf development and allows nutrients from old leaves to be salvaged [73]. SAGs, which are markers of senescence, are upregulated in the senescing leaves of $A$. thaliana [74]. Overexpression of $S A G 29$ has been shown to result in accelerated senescence, and osmotic stresses, such as high salinity, cold, and drought, induce the accumulation of its transcripts [75].

$N Y C 1$ and $N O L$ encode chlorophyll $b$ reductase, which is involved in the biochemical pathway of chlorophyll degradation [76] and accelerates the reduction reaction of chlorophyll $b$ in conversion to chlorophyll $a$ [73, 76]. $\mathrm{PPH}$ is an essential enzyme for chlorophyll breakdown that catalyzes the transformation of pheophytin a (Mgfree chlorophyll $a$ ) into pheophorbide $a$ [77], which is then converted to a red chlorophyll catabolite by PAO [78]. The activity of PAO is modulated by NYE1, which is also known as SGR1 $[79,80]$. In $A$. thaliana, a $P P H-$ deficient mutant (pph-1) exhibits a stay-green phenotype due to the prevention of chlorophyll degradation during senescence [77]. The Atnye1, Atpao1, Osnol1 and Osnyc1 mutants all exhibit a stay-green phenotype under natural and/or induction conditions [73, 76, 79, 81]. The down-regulation of SAGs (12, 13 and 29), NYC1, NOL, $P P H, P A O$ and NYE1/SGR1, in CiWRKY40-4-overexpressing Arabidopsis indicates that CiWRKY40-4 delays Arabidopsis leaf senescence by directly or indirectly down-regulating these genes; however, further investigation will be required to elucidate the mechanism.

Taken together, these results indicate that different CiWRKYs play different roles in plant responses to abiotic stress and developmental processes. Hence, there is an urgent need for examination of the function of CiWRKYs in tolerance against abiotic stresses in $C$. intermedia in future studies.

\section{Conclusions}

In this study, 53 WRKY sequences were retrieved from the drought-treated transcriptome of $C$. intermedia, among which 28 CiWRKYS exhibited full-length sequences. This is the first study to analyze WRKY family genes in C. intermedia based on transcriptome data. The predicted proteins were grouped via phylogenetic tree analysis with WRKYs from A. thaliana and G. max, and their WRKY domains were characterized through multiple sequence alignment of the CiWRKY genes. Furthermore, the subcellular localization of both CiWRKY28-1, with one WRKY domain, and CiWRKY26, with two
WRKY domains, is nuclear. The majority of the 28 CiWRKYs with full-length sequences are expressed in more than one tissue. The expression patterns of the 28 CiWRKYs in response to different abiotic stresses (drought, salt, cold, high-pH and ABA) were examined, and the abiotic stress-responsive genes were further evaluated. Additionally, overexpression of CiWRKY75-1 and CiWRKY40-4 in A. thaliana resulted in hypersensitivity to drought stress and delayed leaf senescence, respectively, compared with the wild type. The results of this study will be useful for understanding the involvement of WRKY genes in stress resistance and plant development and will provide the basis for future functional studies on WRKYs.

\section{Methods}

WRKY identification, phylogenetic analysis and multiple sequence alignment

CiWRKY sequences were derived from a drought-treated RNA-seq database of C. intermedia, in which dehydration treatment was performed by placing the whole seedlings on filter paper for 1,3 or $12 \mathrm{~h}$. The dataset was submitted to NCBI SRA under the accession number SRP121096. The WRKY sequences of $A$. thaliana and G. max were downloaded from the TAIR database (The Arabidopsis Information Resource: http:// www.arabidopsis.org/) and the JGI database (https:// phytozome.jgi.doe.gov), respectively.

The conserved protein domains were subjected to Blast searches against the NCBI database (https://blast.ncbi.nlm.nih.gov/Blast.cgi?PROGRAM=blastx\&PAGE_TYPE=BlastSearch\&LINK_LOC=blasthome) using blastx. After the removal of redundant sequences and WRKY domain prediction, a total of 53 putative CiWRKYs containing the WRKY domain were obtained, which were named according to their similarity with Arabidopsis WRKYs.

A phylogenetic tree of CiWRKYs was constructed using MEGA6.0 and the neighbor-joining (NJ) method, with 1000 bootstrap replications, based on the amino acid sequences of $A$. thaliana and G. $\max$ WRKY proteins.

DNAMAN7 software was used to analyze the core sequences of the WRKY domain via multiple sequence alignment.

\section{Plant materials and abiotic stress treatments}

Seeds from wild $C$. intermedia were collected from Wulanchabu City, Inner Mongolia Autonomous Region, China $(41.44 \mathrm{~N}, 111.69 \mathrm{E})$. No specific field permissions were required to collect the plant samples. The plant material used in this study had been formally identified by Dr. Liwang Qi (Chinese Academy of Forestry), Prof. Ling Yan (Inner Mongolia Agricultural University) and Prof. Meng Ji (Inner Mongolia Academy of Forestry). 
The seeds were sown in pots containing peat soil and vermiculite $(1: 2 \mathrm{v} / \mathrm{v})$ under long-day conditions (16-hlight/8-h-dark cycle) at $22{ }^{\circ} \mathrm{C}$. One-month-old plants were employed for the abiotic stress treatments, as previously described [51, 82]. Briefly, prior to the drought, salt, high-pH (10) and ABA treatments, whole seedlings were removed from soil and cleaned with tap water. For drought treatment, whole seedlings were placed on filter paper for $0.5,1,3,6,12,24$ or $48 \mathrm{~h}$ at room temperature. For the salt, high-pH (10) and ABA treatments, the roots of the seedlings were soaked with solutions containing $300 \mathrm{mM} \mathrm{NaCl}, 200 \mathrm{mM} \mathrm{NaHCO}$ (using $\mathrm{NaOH}$ to adjust the $\mathrm{pH}$ to 10 ) or $100 \mu \mathrm{M}$ ABA, respectively, for $0.5,1,3,6,12,24$ or $48 \mathrm{~h}$. For cold treatment, pots containing seedlings were transferred to $4{ }^{\circ} \mathrm{C}$ and then cultured for $0.5,1,3,6,12,24$ or $48 \mathrm{~h}$. Untreated plants were used as controls. At each time point in each treatment, the shoots of three plants were harvested as one sample; each plant was only used once for tissue harvesting, at one time point, and was not subjected to any further treatment thereafter. Samples were snap frozen in liquid nitrogen and stored at $-80{ }^{\circ} \mathrm{C}$ until they were employed for total RNA extraction. All experiments were repeated three times.

Additionally, the roots, stems, and leaves of wild $C$. intermedia were collected during the flowering stage for tissue-specific expression analysis.

The leaves of four-week-old wild-type and transgenic Arabidopsis were used for relative gene expression analysis.

\section{PRO, SS and MDA measurements}

One-month-old $C$. intermedia seedlings cultured in growth chambers were subjected to drought treatment, and physiological indices were measured at different time points $(0,1,3,6,12,24$ or $48 \mathrm{~h})$. The contents of PRO, MDA and SS were determined in colorimetric assays, using the ninhydrin coloration, thiobarbituric acid and anthrone methods, respectively [83].

\section{Total RNA extraction and CDNA synthesis}

Total RNA was isolated from samples using the TRIzol regent. For tissue-specific expression, RNA was extracted from different tissues, including the roots, stems, and leaves. For the examination of abiotic stressresponsive expression, RNA was extracted from shoots. For gene expression analysis in Arabidopsis, RNA was extracted from leaves. RNA integrity was examined through agarose gel electrophoresis, and RNA purity was determined based on the $A_{260} \mathrm{~nm} / \mathrm{A}_{280 \mathrm{~nm}}$ and $\mathrm{A}_{260 \mathrm{~nm}} / \mathrm{A}_{230 \mathrm{~nm}}$ ratios.

After pretreatment with RNase-free DNase I (Takara, Dalian, China), total RNA $(1 \mu \mathrm{g})$, was used to synthesize first-strand cDNA employing an M-MLV reverse transcriptase kit (Takara) according to the manufacturer's instructions. The cDNA was then diluted 16-fold to be employed as a template for qRT-PCR analysis.

\section{qRT-PCR analysis of gene expression}

qRT-PCR was performed using SYBR Premix Ex Taq II (Takara) on a LightCycler 480 Real Time PCR system (Roche, Basel, Switzerland), as previously described [84]. The thermal cycling program was as follows: $95{ }^{\circ} \mathrm{C}$ for $30 \mathrm{~s}$, followed by 40 cycles of $95{ }^{\circ} \mathrm{C}$ for $5 \mathrm{~s}, 60{ }^{\circ} \mathrm{C}$ for $30 \mathrm{~s}$ and $72{ }^{\circ} \mathrm{C}$ for $15 \mathrm{~s}$. CiEF1 $\alpha$ (GenBank: KC679842) [51] was employed as a reference gene to normalize target gene expression levels in the $C$. intermedia samples, and the $2^{-\Delta \Delta C T}$ and $2^{-\Delta C T}$ methods [85] were used to calculate the relative expression levels of stress-responsive and

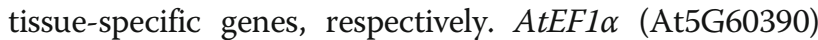
[84] was employed as a reference gene for the normalization of target gene expression levels in Arabidopsis samples via the $2^{-\Delta \Delta C T}$ method [85]. Three technical replicates for each reaction were performed. The primers employed for qRT-PCR analysis are listed in Additional file 8: Table S3.

\section{CiWRKY transgenic A. thaliana}

To generate the recombinant CiWRKY overexpression vector, the full-length CDSs of CiWRKY75-1 and CiWRKY40-4 were amplified using PCR product from wild $C$. intermedia cDNA and cloned into the expression vector pCanG-HA using the restriction enzymes SalI/ SpeI and SacI/SalI, respectively.

To generate the recombinant CiWRKY and GFP-infused vectors, the coding sequences of CiWRKY26 and CiWRKY28-1 without the stop codon were amplified using PCR, and the PCR products were then cloned into the $\mathrm{N}$ - terminus of the GFP-encoding sequence in the pCAMBIA1302 vector driven by the cauliflower mosaic virus (CaMV) $35 \mathrm{~S}$ promoter. The primers used for the construction of all of the recombination vectors are listed in Additional file 8: Table S3.

The recombinant vectors were expressed in wild-type A. thaliana using the floral dipping method [86], mediated by Agrobacterium tumefaciens (strain GV3101). The empty vector was used as the control.

\section{Subcellular localization of CiWRKYs}

The roots of 10-day-old $\mathrm{T}_{2}$-generation transgenic seedlings were employed for observations of GFP fluorescence under an LSM510 confocal laserscanning microscope (Carl Zeiss), with $488 \mathrm{~nm}$ argon excitation and a $505-530 \mathrm{~nm}$ band filter, as previously described [84]. 


\section{Drought tolerance test}

To assess the potential drought tolerance of the CiWRKY-overexpressing lines, four-week-old transgenic plants and wild-type plants growing in the same pots were exposed to drought stress by withholding water for 15 days, and the plants were then re-watered and allowed to grow for an additional 3 days. Survival rates were calculated using 30 plants per genotype for each experiment. The leaf samples were harvested before or immediately after drought treatment to measure physiological indices. This experiment was repeated three times with similar results.

\section{Additional files}

Additional file 1: Table S1. Expression data for CiWRKY genes in different $C$. intermedia tissues. The expression data were generated via qRT-PCR, and calculations were performed using the $2^{-\Delta C T}$ method; CiEF1a was used as an endogenous control. (XLS $22 \mathrm{~kb}$ )

Additional file 2: Figure S1. Changes in physiological activity in $C$. intermedia under drought treatment. The abscissa indicates the time points in the treatments, and the ordinate indicates physiological activities. (a) MDA content. (b) Pro content. (c) SS content. (PDF $112 \mathrm{~kb}$ )

Additional file 3: Figure S2. Expression patterns of CiWRKYs under salt treatment. Samples were collected from the shoots of one-month-old C. intermedia seedlings at $0.5,1,3,6,12,24$ or $48 \mathrm{~h}$ following salt treatment, and untreated plants were employed as the control. The expression levels of 28 CiWRKYs with full-length sequences were examined via qRT-PCR. Expression values were estimated using the $2^{-\Delta \Delta C T}$ method, and CiEFla was used as reference gene. The error bars represent the means of three technical replicates \pm SD. (PDF $258 \mathrm{~kb}$ )

Additional file 4: Figure S3. Expression patterns of CiWRKYs under ABA treatment. Samples were collected from the shoots of one-month-old C. intermedia seedlings at $0.5,1,3,6,12,24$ or $48 \mathrm{~h}$ following $A B A$ treatment, and untreated plants were employed as the control. The expression levels of 28 CiWRKYs with full-length sequences were examined via GRT-PCR. Expression values were estimated using the $2^{-\Delta \Delta C T}$ method, and CiEFla was used as reference gene. The error bars represent the means of three technical replicates \pm SD. (PDF $254 \mathrm{~kb}$ )

Additional file 5: Figure S4. Expression patterns of CiWRKYs under cold treatment. Samples were collected from the shoots of one-month-old C. intermedia seedlings at $0.5,1,3,6,12,24$ or $48 \mathrm{~h}$ following cold treatment, and untreated plants were employed as the control. The expression levels of 28 CiWRKYs with full-length sequences were examined via qRT-PCR. Expression values were estimated using the $2^{-\Delta \Delta C T}$ method, and CiEFla was used as reference gene. The error bars represent the means of three technical replicates \pm SD. (PDF $253 \mathrm{~kb}$ )

Additional file 6: Figure S5. Expression patterns of CiWRKYs under high-pH treatment. Samples were collected from the shoots of one-month-old C. intermedia seedlings at $0.5,1,3,6,12,24$ or $48 \mathrm{~h}$ following high-pH treatment, and untreated plants were employed as the control. The expression levels of 28 CiWRKYs with full-length sequences were examined via qRT-PCR. Expression values were estimated using the $2^{-\Delta \Delta C T}$ method, and CiEFla was used as reference gene. The error bars represent the means of three technical replicates \pm SD. (PDF $257 \mathrm{~kb}$ )

Additional file 7: Table S2. Expression data for 28 CiWRKY genes with complete ORFs based on drought-treated transcriptome data. (XLS $22 \mathrm{~kb}$ )

Additional file 8: Table S3. Primers employed for qRT-PCR and the analysis of transgenic plant construction. (XLS $27 \mathrm{~kb}$ )

\section{Abbreviations}

ABA: Abscisic acid; GFP: Green fluorescent protein; MDA: Malondialdehyde NOL: NYC1-like; NYC1: Non-yellow coloring1; NYE1/SGR1: Non-yellowing1/staygreen 1; OE: Overexpression; ORF: Open reading frame; PAO: Pheophorbide a oxygenase; PPH: Pheophytinase; PRO: Proline; qRT-PCR: Quantitative real-time PCR; RACE: Rapid-amplification of cDNA ends; SS: Soluble total sugar

\section{Acknowledgements}

We thank Dr. Yan Wang for critical reading the manuscript, and thank Nature Research Editing Service for language editing.

\section{Funding}

This work was supported by National Natural Science Foundation of China (No.31360169), and Inner Mongolia Science \& Technology Plan (201503004). The funding bodies only provide the financial support in the design of the study and collection, analysis, and interpretation of data and in editing the manuscript.

\section{Availability of data and materials}

The transcriptome clean raw reads data that support the findings of this study have been deposited in NCBI Sequence Read Archive (https:// www.ncbi.nlm.nih.gov/Traces/sra_sub/) under the accession number SRP121096. The sequence of CiWRKYS analyzed during the current study is available from the corresponding author on reasonable request. Other relevant data analyzed during this study are included in this published article and its supplementary information files.

\section{Authors' contributions}

RW, GL and YW conceived and designed the study. YW and MM performed the experiments. YW and DW integrated and analyzed the data. YW, MM and DW prepared the figures; $\mathrm{QY}, \mathrm{FY}$ and $\mathrm{MD}$ participated in the design of the experiments. DW and YW wrote the manuscript, and GL revised the manuscript. All authors read and approved the final manuscript.

Ethics approval and consent to participate

Not applicable.

Consent for publication

Not applicable.

\section{Competing interests}

The authors declare that they have no competing interests.

\section{Publisher's Note}

Springer Nature remains neutral with regard to jurisdictional claims in published maps and institutional affiliations.

\section{Author details}

${ }^{1}$ College of Life Sciences, Inner Mongolia Key Laboratory of Plant Stress Physiology and Molecular Biology, Inner Mongolia Agricultural University, Hohhot, China. Institute of Grassland Research, Chinese Academy of Agricultural Sciences, Key Laboratory of Grassland Ecology and Restoration, Ministry of Agriculture, Hohhot, China. ${ }^{3}$ College of Food Science and Engineering, Inner Mongolia Agricultural University, Hohhot, China.

Received: 11 July 2017 Accepted: 14 January 2018

Published online: 09 February 2018

\section{References}

1. Li C, Li D, Shao F, Molecular LS. Cloning and expression analysis of WRKY transcription factor genes in salvia miltiorrhiza. BMC Genomics. 2015;16(1):200.

2. Singh K, Foley RC, Onate-Sanchez L. Transcription factors in plant defense and stress responses. Curr Opin Plant Biol. 2002;5(5):430-6.

3. Eulgem T, Rushton PJ, Robatzek S, Somssich IE. The WRKY superfamily of plant transcription factors. Trends Plant Sci. 2000;5(5):199-206.

4. Ulker B, Somssich IE. WRKY transcription factors: from DNA binding towards biological function. Curr Opin Plant Biol. 2004;7(5):491-8.

5. Rushton PJ, Somssich IE, Ringler P, Shen QJ. WRKY transcription factors. Trends Plant Sci. 2010;15(5):247-58. 
6. Dong J, Chen C, Expression CZ. Profiles of the Arabidopsis WRKY gene superfamily during plant defense response. Plant Mol Biol. 2003;51(1):21-37.

7. Bencke-Malato M, Cabreira C, Wiebke-Strohm B, Bucker-Neto L, Mancini E, Osorio MB, et al. Genome-wide annotation of the soybean WRKY family and functional characterization of genes involved in response to Phakopsora pachyrhizi infection. BMC Plant Biol. 2014;14(1):236.

8. Meng D, Li Y, Bai Y, Li M, Cheng L. Genome-wide identification and characterization of WRKY transcriptional factor family in apple and analysis of their responses to waterlogging and drought stress. Plant physiology and biochemistry : PPB/Societe francaise de physiologie vegetale. 2016;103:71-83.

9. Zhang Y, Feng JC. Identification and characterization of the grape WRKY family. Biomed Res Int 2014;2014:787680.

10. Wen F, Zhu H, Li P, Jiang M, Mao W, Ong C, et al. Genome-wide evolutionary characterization and expression analyses of WRKY family genes in Brachypodium Distachyon. DNA Res. 2014;21 (3):327-39.

11. Wei Y, Shi H, Xia Z, Tie W, Ding Z, Yan Y, et al. Genome-wide identification and expression analysis of the WRKY gene family in cassava. Front Plant Sci. $2016 ; 7(25)$

12. Li MY, Xu ZS, Tian C, Huang Y, Wang F, Xiong AS. Genomic identification of WRKY transcription factors in carrot (Daucus Carota) and analysis of evolution and homologous groups for plants. Sci Rep. 2016:6(23101)

13. Ramamoorthy R, Jiang SY, Kumar N, Venkatesh PN, Ramachandran S. A comprehensive transcriptional profiling of the WRKY gene family in rice under various abiotic and phytohormone treatments. Plant Cell Physiol. 2008:49(6):865-79.

14. Yue H, Wang M, Liu S, Du X, Song W, Nie X. Transcriptome-wide identification and expression profiles of the WRKY transcription factor family in broomcorn millet (Panicum Miliaceum L.). BMC Genomics. 2016;17(343)

15. Wei KF, Chen J, Chen YF, Wu L, Xie DX. Molecular phylogenetic and expression analysis of the complete WRKY transcription factor family in maize. DNA Res. 2012;19(2):153-64.

16. Dou L, Zhang X, Pang C, Song M, Wei H, Fan S, et al. Genome-wide analysis of the WRKY gene family in cotton. Mol Gen Genomics. 2014;289(6):1103-21.

17. Luo M, Dennis ES, Berger F, Peacock WJ, Chaudhury A. MINISEED3 (MINI3), a WRKY family gene, and HAIKU2 (IKU2), a leucine-rich repeat (LRR) KINASE gene, are regulators of seed size in Arabidopsis. P Natl Acad Sci USA. 2005;102(48):17531-6.

18. Zhang CQ, Xu Y, Lu Y, Yu HX, Gu MH, Liu OQ. The WRKY transcription factor OsWRKY78 regulates stem elongation and seed development in rice. Planta. 2011;234(3):541-54.

19. Ding ZJ, Yan JY, Li GX, Wu ZC, Zhang SQ, Zheng SJ. WRKY41 controls Arabidopsis seed dormancy via direct regulation of ABI3 transcript levels not downstream of ABA. Plant J. 2014;79(5):810-23.

20. Jiang W, Yu D. Arabidopsis WRKY2 transcription factor mediates seed germination and postgermination arrest of development by abscisic acid. BMC Plant Biol. 2009;9(1):96.

21. Johnson CS, Kolevski B, Smyth DR. TRANSPARENT TESTA GLABRA2, a trichome and seed coat development gene of Arabidopsis, encodes a WRKY transcription factor. Plant Cell. 2002;14(6):1359-75.

22. Grunewald W, De Smet I, Lewis DR, Lofke C, Jansen L, Goeminne G, et al. Transcription factor WRKY23 assists auxin distribution patterns during Arabidopsis root development through local control on flavonol biosynthesis. Proc Natl Acad Sci U S A. 2012:109(5):1554-9.

23. Miao Y, Laun T, Zimmermann P, Zentgraf U. Targets of the WRKY53 transcription factor and its role during leaf senescence in Arabidopsis. Plant Mol Biol. 2004;55(6):853-67.

24. Li W, Wang H, Yu D. Arabidopsis WRKY transcription factors WRKY12 and WRKY13 oppositely regulate flowering under short-day conditions. Mol Plant. 2016;9(11):1492-503.

25. Yang Y, Chi Y, Wang Z, Zhou Y, Fan B, Chen Z. Functional analysis of structurally related soybean GmWRKY58 and GmWRKY76 in plant growth and development. J Exp Bot. 2016;67(15):4727-42.

26. Pandey SP, Somssich IE. The role of WRKY transcription factors in plant immunity. Plant Physiol. 2009;150(4):1648-55.

27. Liu J, Chen X, Liang X, Zhou X, Yang F, He SY, et al. Alternative splicing of Rice WRKY62 and WRKY76 transcription factor genes in pathogen defense. Plant Physiol. 2016;171(2):1427-42.

28. Qiu D, Xiao J, Ding X, Xiong M, Cai M, Cao Y, et al. OsWRKY13 mediates rice disease resistance by regulating defense-related genes in salicylate- and jasmonate-dependent signaling. Mol Plant-Microbe Interact. 2007;20(5):492-9.
29. Tao Z, Liu H, Qiu D, Zhou Y, Li X, Xu C, et al. A pair of allelic WRKY genes play opposite roles in rice-bacteria interactions. Plant Physiol. 2009;151(2):936-48.

30. Cheng HT, Liu HB, Deng Y, Xiao JH, Li XH, Wang SP. The WRKY45-2 WRKY13 WRKY42 transcriptional regulatory Cascade is required for Rice resistance to fungal pathogen. Plant Physiol. 2015;167(3):1087-99.

31. Yu F, Huaxia Y, Lu W, Wu C, Cao X, Guo X. GhWRKY15, a member of the WRKY transcription factor family identified from cotton (Gossypium Hirsutum L.), is involved in disease resistance and plant development. BMC Plant Biol. 2012;12(1):144.

32. Marchive C, Mzid R, Deluc L, Barrieu F, Pirrello J, Gauthier A, et al. Isolation and characterization of a Vitis Vinifera transcription factor, VVWRKY1, and its effect on responses to fungal pathogens in transgenic tobacco plants. J Exp Bot. 2007;58(8):1999-2010.

33. Luo X, Bai X, Sun X, Zhu D, Liu B, Ji W, et al. Expression of wild soybean WRKY20 in Arabidopsis enhances drought tolerance and regulates ABA signalling. J Exp Bot. 2013;64(8):2155-69.

34. Jiang Y, Liang G, Yu D. Activated expression of WRKY57 confers drought tolerance in Arabidopsis. Mol Plant. 2012;5(6):1375-88.

35. Sun J, Hu W, Zhou R, Wang L, Wang X, Wang Q, et al. The Brachypodium Distachyon BdWRKY36 gene confers tolerance to drought stress in transgenic tobacco plants. Plant Cell Rep. 2015;34(1):23-35.

36. Ren $X$, Chen Z, Liu Y, Zhang H, Zhang $M$, Liu Q, et al. ABO3, a WRKY transcription factor, mediates plant responses to abscisic acid and drought tolerance in Arabidopsis. Plant J. 2010;63(3):417-29.

37. Tao Z, Kou Y, Liu H, Li X, Xiao J, Wang S. OsWRKY45 alleles play different roles in abscisic acid signalling and salt stress tolerance but similar roles in drought and cold tolerance in rice. J Exp Bot. 2011;62(14):4863-74.

38. Li S, Zhou X, Chen L, Huang W, Yu D. Functional characterization of Arabidopsis Thaliana WRKY39 in heat stress. Mol Cells. 2010;29(5):475-83.

39. Li S, Fu Q, Huang W, Yu D. Functional analysis of an Arabidopsis transcription factor WRKY25 in heat stress. Plant Cell Rep. 2009;28(4):683-93.

40. Chen YF, Li LQ, Xu Q, Kong YH, Wang H, Wu WH. The WRKY6 transcription factor modulates PHOSPHATE1 expression in response to low pi stress in Arabidopsis. Plant Cell. 2009;21(11):3554-66

41. Dai X, Wang Y, Zhang WH. OsWRKY74, a WRKY transcription factor, modulates tolerance to phosphate starvation in rice. J Exp Bot. 2016:67(3):947-60.

42. Wang H, Hao J, Chen X, Hao Z, Wang X, Lou Y, et al. Overexpression of rice WRKY89 enhances ultraviolet $B$ tolerance and disease resistance in rice plants. Plant Mol Biol. 2007;65(6):799-815.

43. Li J, Besseau S, Toronen P, Sipari N, Kollist H, Holm L, et al. Defense-related transcription factors WRKY70 and WRKY54 modulate osmotic stress tolerance by regulating stomatal aperture in Arabidopsis. New Phytol. 2013;200(2):457-72

44. Chen H, Lai Z, Shi J, Xiao Y, Chen Z, Xu X. Roles of arabidopsis WRKY18, WRKY40 and WRKY60 transcription factors in plant responses to abscisic acid and abiotic stress. BMC Plant Biol. 2010;10(1):281.

45. Xu ZZ, Zhou GS, Wang YH. Combined effects of elevated CO2 and soil drought on carbon and nitrogen allocation of the desert shrub Caragana Intermedia. Plant Soil. 2007:301(1-2):87-97.

46. Wu BF, Li WF, Xu HY, Qi LW, Han SY. Role of cin-miR2118 in drought stress responses in Caragana Intermedia and tobacco. Gene. 2015;574(1):34-40.

47. Guo WH, Li B, Zhang XS, Wang RQ. Effects of water stress on water use efficiency and water balance components of Hippophae Rhamnoides and Caragana Intermedia in the soil-plant-atmosphere continuum. Agrofor Syst. 2010;80(3):423-35.

48. Shi SQ, Shi Z, Jiang ZP, Qi LW, Sun XM, Li CX, et al. Effects of exogenous GABA on gene expression of Caragana Intermedia roots under $\mathrm{NaCl}$ stress: regulatory roles for $\mathrm{H} 2 \mathrm{O} 2$ and ethylene production. Plant Cell Environ. 2010;33(2):149-62.

49. Zhu JF, Li WF, Yang WH, Qi LW, Han SY. Identification of microRNAs in Caragana Intermedia by high-throughput sequencing and expression analysis of 12 microRNAs and their targets under salt stress. Plant Cell Rep. 2013;32(9):1339-49.

50. Zhu JF, Zhang LF, Li WF, Han SY, Yang WH, Qi LW. Reference gene selection for quantitative real-time PCR normalization in Caragana Intermedia under different abiotic stress conditions. PLoS One. 2013:8(1):e53196.

51. Han X, Feng Z, Xing D, Yang Q, Wang R, Qi L, et al. Two NAC transcription factors from Caragana Intermedia altered salt tolerance of the transgenic Arabidopsis. BMC Plant Biol. 2015;15(1):208. 
52. Ji J, Zheng L, Yue J, Yao X, Chang E, Xie T, et al. Identification of two CiGADs from Caragana Intermedia and their transcriptional responses to abiotic stresses and exogenous abscisic acid. PeerJ. 2017;5:e3439.

53. Guo C, Guo R, Xu X, Gao M, Li X, Song J, et al. Evolution and expression analysis of the grape (Vitis Vinifera L.) WRKY gene family. J Exp Bot. 2014;65(6):1513-28.

54. Mohanta TK, Park YH, Bae H. Novel genomic and evolutionary insight of WRKY transcription factors in plant lineage. Sci Rep. 2016;6:37309.

55. Marchler-Bauer A, Derbyshire MK, Gonzales NR, Lu S, Chitsaz F, Geer LY, et al. CDD: NCBI's conserved domain database. Nucleic Acids Res. 2015:43(Database issue):D222-6.

56. Hu W, Wang LZ, Tie WW, Yan Y, Ding ZH, Liu JH, et al. Genome-wide analyses of the bZIP family reveal their involvement in the development, ripening and abiotic stress response in banana. Sci Rep. 2016;6:30203.

57. Jakoby M, Weisshaar B, Droge-Laser W, Vicente-Carbajosa J, Tiedemann J, Kroj T, et al. bZIP transcription factors in Arabidopsis. Trends Plant Sci. 2002;7(3):106-11

58. Zhao TT, Zhang J, Liang LS, Ma QH, Chen X, Zong JW, et al. Expression and functional analysis of WRKY transcription factors in Chinese wild hazel, Corylus Heterophylla Fisch. PLoS One. 2015;10(8):e0135315.

59. Fan X, Guo Q, Xu P, Gong Y, Shu H, Yang Y, et al. Transcriptome-wide identification of salt-responsive members of the WRKY gene family in Gossypium Aridum. PLoS One. 2015;10(5):e0126148.

60. Xiong L, Schumaker KS, Zhu JK. Cell signaling during cold, drought, and salt stress. Plant Cell. 2002;14(Suppl):S165-83.

61. Sun YD, Yu DQ. Activated expression of AtWRKY53 negatively regulates drought tolerance by mediating stomatal movement. Plant Cell Rep. 2015;34(8):1295-306

62. He GH, Xu JY, Wang YX, Liu JM, Li PS, Chen M, et al. Drought-responsive WRKY transcription factor genes TaWRKY1 and TaWRKY33 from wheat confer drought and/or heat resistance in Arabidopsis. BMC Plant Biol. 2016;16(1):116.

63. Devaiah BN, Karthikeyan AS, Raghothama KG. WRKY75 transcription factor is a modulator of phosphate acquisition and root development in arabidopsis. Plant Physiol. 2007;143(4):1789-801.

64. Encinas-Villarejo S, Maldonado AM, Amil-Ruiz F, de los Santos B, Romero F, Pliego-Alfaro $F$, et al. Evidence for a positive regulatory role of strawberry (Fragaria $x$ ananassa) fa WRKY1 and Arabidopsis at WRKY75 proteins in resistance. J Exp Bot. 2009;60(11):3043-65.

65. Chen X, Liu J, Lin G, Wang A, Wang Z, Lu G. Overexpression of AtWRKY28 and AtWRKY75 in Arabidopsis enhances resistance to oxalic acid and Sclerotinia sclerotiorum. Plant Cell Rep. 2013;32(10):1589-99.

66. Ding ZJ, Yan JY, Xu XY, Yu DQ, Li GX, Zhang SQ, et al. Transcription factor WRKY46 regulates osmotic stress responses and stomatal movement independently in Arabidopsis. Plant J. 2014;79(1):13-27.

67. Chen J, Nolan TM, Ye H, Zhang M, Tong H, Xin P, et al. Arabidopsis WRKY46, WRKY54, and WRKY70 transcription factors are involved in Brassinosteroidregulated plant growth and drought responses. Plant Cell. 2017;29(6):1425-39.

68. Xu XP, Chen CH, Fan BF, Chen ZX. Physical and functional interactions between pathogen-induced Arabidopsis WRKY18, WRKY40, and WRKY60 transcription factors. Plant Cell. 2006;18(5):1310-26.

69. Pandey SP, Roccaro M, Schon M, Logemann E, Somssich IE. Transcriptional reprogramming regulated by WRKY18 and WRKY40 facilitates powdery mildew infection of Arabidopsis. Plant J. 2010;64(6):912-23.

70. Shang Y, Yan L, Liu ZQ, Cao Z, Mei C, Xin Q, et al. The mg-chelatase H subunit of Arabidopsis antagonizes a group of WRKY transcription repressors to relieve ABA-responsive genes of inhibition. Plant Cell. 2010;22(6):1909-35.

71. Lee MH, Jeon HS, Kim HG, Park OK. An Arabidopsis NAC transcription factor NAC4 promotes pathogen-induced cell death under negative regulation by microRNA164. New Phytol. 2017;214(1):343-60.

72. Zhang S, Li C, Wang R, Chen Y, Shu S, Huang R, et al. The Arabidopsis mitochondrial protease $\mathrm{FtSH} 4$ is involved in leaf senescence via regulation of WRKY-dependent salicylic acid accumulation and signaling. Plant Physiol. 2017;173(4):2294-307.

73. Kusaba M, Ito H, Morita R, lida S, Sato Y, Fujimoto M, et al. Rice NONYELLOW COLORING1 is involved in light-harvesting complex II and grana degradation during leaf senescence. Plant Cell. 2007:19(4):1362-75.

74. Lohman KN, SG MCJ, Amasino RM. Molecular analysis of natural leaf senescence in Arabidopsis Thaliana. Physiol Plant. 1994;92(2):322-8.
75. Seo PJ, Park JM, Kang SK, Kim SG, Park CM. An Arabidopsis senescenceassociated protein SAG29 regulates cell viability under high salinity. Planta. 2011;233(1):189-200

76. Sato Y, Morita R, Katsuma S, Nishimura M, Tanaka A, Kusaba M. Two shortchain dehydrogenase/reductases, NON-YELLOW COLORING 1 and NYC1LIKE, are required for chlorophyll b and light-harvesting complex \|| degradation during senescence in rice. Plant J. 2009;57(1):120-31.

77. Schelbert S, Aubry S, Burla B, Agne B, Kessler F, Krupinska K, et al. Pheophytin pheophorbide hydrolase (pheophytinase) is involved in chlorophyll breakdown during leaf senescence in Arabidopsis. Plant Cell. 2009;21(3):767-85.

78. Qiu K, Li Z, Yang Z, Chen J, Wu S, Zhu X, et al. EIN3 and ORE1 accelerate Degreening during ethylene-mediated leaf senescence by directly activating chlorophyll catabolic genes in Arabidopsis. PLoS Genet. 2015:11(7):e1005399.

79. Ren G, An K, Liao Y, Zhou X, Cao Y, Zhao H, et al. Identification of a novel chloroplast protein AtNYE1 regulating chlorophyll degradation during leaf senescence in Arabidopsis. Plant Physiol. 2007;144(3):1429-41.

80. Sakuraba Y, Schelbert S, Park SY, Han SH, Lee BD, Andres CB, et al. STAYGREEN and chlorophyll catabolic enzymes interact at light-harvesting complex II for chlorophyll detoxification during leaf senescence in Arabidopsis. Plant Cell. 2012;24(2):507-18.

81. Pruzinska A, Tanner G, Aubry S, Anders I, Moser S, Muller T, et al. Chlorophyll breakdown in senescent Arabidopsis leaves. Characterization of chlorophyll catabolites and of chlorophyll catabolic enzymes involved in the degreening reaction. Plant Physiol. 2005;139(1):52-63.

82. Yang Q, Yin J, Li G, Qi L, Yang F, Wang R, et al. Reference gene selection for qRT-PCR in Caragana Korshinskii Kom. Under different stress conditions. Mol Biol Rep. 2014;41(4):2325-34.

83. Shi H, Wang Y, Cheng Z, Ye T, Chan Z. Analysis Of natural variation in bermudagrass (Cynodon Dactylon) reveals physiological responses underlying drought tolerance. PLoS One. 2012;7(12):28.

84. Wan DL, Li RL, Zou B, Zhang X, Cong JY, Wang RG, et al. Calmodulinbinding protein CBP60g is a positive regulator of both disease resistance and drought tolerance in Arabidopsis. Plant Cell Rep. 2012;31(7):1269-81.

85. Livak KJ, Schmittgen TD. Analysis of relative gene expression data using real-time quantitative PCR and the 2(-Delta Delta $C T)$ method. Methods. 2001;25(4):402-8.

86. Clough SJ, Bent AF. Floral dip: a simplified method for agrobacteriummediated transformation of Arabidopsis Thaliana. Plant J. 1998;16(6):735-43.

\section{Submit your next manuscript to BioMed Central and we will help you at every step:}

- We accept pre-submission inquiries

- Our selector tool helps you to find the most relevant journal

- We provide round the clock customer support

- Convenient online submission

- Thorough peer review

- Inclusion in PubMed and all major indexing services

- Maximum visibility for your research

Submit your manuscript at www.biomedcentral.com/submit
C) Biomed Central 